\title{
Social impact of paediatric tracheostomy: our experiences at a tertiary care teaching hospital
}

\author{
Santosh Kumar Swain, Satyabrata Acharya \\ Department of Otorhinolaryngology, IMS and SUM Hospital, Siksha "O" Anusandhan University, K8, Kalinganagar, \\ Bhubaneswar, Odisha, India
}

\section{ABSTRACT}

Introduction: Paediatric patients may require tracheostomy in certain clinical conditions with upper airway obstruction or in critical conditions requiring prolonged ventilation. It is often associated with higher rates of morbidity along with social stigma if it is sustained for a longer period. Although there are several studies available in medical literature for indications, complications, and different techniques for tracheostomy, but most of them do not provide any insight into the social impact of tracheostomy. The aim of this study is to assess the social impact of tracheostomy at a tertiary care teaching hospital.

Material and methods: This is a retrospective observational study done at a tertiary care teaching hospital. The study was carried out from December 2017 to January 2020. Twenty-four paediatric tracheostomy patients participated in this study. The observed complications in tracheostomy such as surgical/medical and social issues were documented. We conducted structured interviews with caregivers of patients with tracheostomy. Results: Medical or surgical complications included excess mucus production (70.83\%), infections at the stoma site (33.33\%), blockage (25\%), granulations around the stoma (16.66\%), and maggots at the stoma (4.16\%). The social impacts on tracheostomy patients included family reluctance to accept the patient with a tracheostomy tube, inability to communicate, and problems with social integration.

Conclusions: Tracheostomy has several ranges of effects on the quality of life of caregivers and patients with tracheostomy. Management of social stigma associated with permanent tracheostomy is more challenging than any complications due to the tracheostomy procedure itself. It can be solved with proper counselling with family members and those involved with patients.

\section{KEY WORDS:}

tracheostomy, social impact, family reluctance, quality of life.

\section{INTRODUCTION}

Tracheostomy is one of the ancient surgical procedures, in which an opening is made in the anterior wall of the trachea, converting it into a stoma with the skin surface of the neck [1]. The indications of the tracheostomy and its consequences among the paediatric age group have changed over the years [2]. Initially, acute infections such as epiglottitis or diphtheria were important indications of tracheostomy in children. The development and spread of vaccines for Corynebacterium diphtheriae and Haemophilus influenzae along with use of endotracheal intubation have decreased the infection-based indications of tracheostomy.

\section{ADDRESS FOR CORRESPONDENCE:}

Santosh Kumar Swain, Department of Otorhinolaryngology, IMS and SUM Hospital, Siksha "O" Anusandhan

University, K8, Kalinganagar, 751003 Bhubaneswar, Odisha, India, ORCID: 0000-0001-7933-4414,

e-mail: santoshvoltaire@yahoo.co.in 
Tracheostomy may be of temporary or permanent type. In temporary tracheostomy, the tracheostomy tube stays in place for a few days to a few weeks and is finally removed with the resolution of the primary disease or when the airway obstruction is cleared. Once the tracheostomy tube is removed, the stoma will be closed spontaneously by using an airtight dressing or surgical closure. A permanent tracheostomy is usually left in the patient for as long as he or she lives. This permanent tracheostomy is due to irreversible injury to the laryngeal architecture or bilateral recurrent laryngeal nerves, which make the larynx inadequate for breathing through the laryngeal inlet. Indications for permanent tracheostomy include subglottic stenosis, total laryngectomy, bilateral recurrent laryngeal nerve paralysis or collapse, laryngotracheal resection, and staged laryngeal reconstruction [3]. Permanent tracheostomy is also done in the case of severe obstructive sleep apnoea syndrome in which the patient cannot tolerate continuous positive airway pressure and other surgical procedures for sleep apnoea syndrome have failed [4].

Permanent tracheostomy affects vocal communication, eating, water-based activities, sleeping, and leisure activities of the person in daily life. Children with a tracheostomy tube must be supervised at all times by parents or care givers. Parents or caregivers respond to all the child's needs as well as emergency conditions. Parents and caregivers often bear mental and physical stress during care. In the case of permanent tracheostomy, the constant presence of the tube in situ leads to undesirable effects to the neck tissue. There are very few published data regarding the social impact of paediatric tracheostomy, whereas

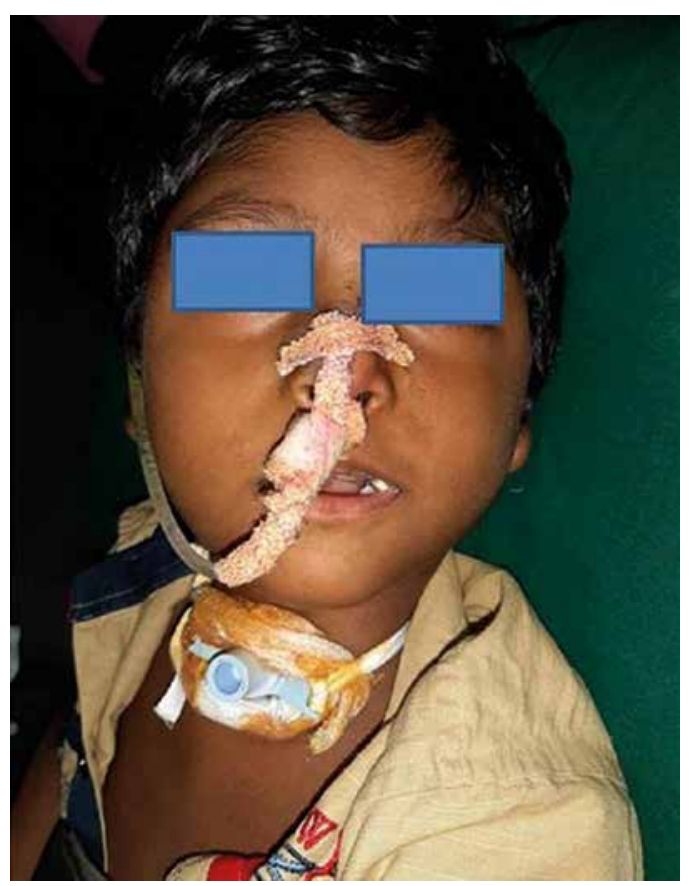

FIGURE 1. A 3-year-old boy who underwent tracheostomy for stridor (bilateral abductor palsy). Later his parents were reluctant to discharge him from the hospital several data exist on the changing scenario of different surgical techniques, indications, and complications of this surgery, which does not offer any insight into the social impact of tracheostomy. In this study, we analyse the social impact of paediatric permanent tracheostomy at a tertiary care teaching hospital in eastern India.

\section{MATERIAL AND METHODS}

This study is retrospective and observational, carried out at our Institute for a period of three years. The purpose of this study was explained to all the parents/caregivers of the children with tracheostomy tubes. This study was approved by the Institutional Ethics Committee (IEC), and the study was carried out on 24 patients attending the Outpatient Department of Otorhinolaryngology. The study was carried out from December 2017 to January 2020. All the parents/caregivers of paediatric patients of this study how came to the outpatient department for tracheostomy care were interviewed. The age ranges of the participants were 3-18 years. Both genders participated in this study, with 15 male and 9 female. All of them underwent tracheostomy during last one year. Out of 24 patients, 16 underwent tracheostomy at our institute, and the rest were done elsewhere. All the paediatric patients who participated in this study had undergone fibreoptic laryngoscopy for assessment of laryngeal pathology. The inclusion criterion for this study was any child with a tracheostomy in situ for more than one year. We targeted all the caregivers and parents of children with tracheostomy attending the Outpatient Department of Tracheostomy for care and decannulation. Interviews were done face to face with parents, caregivers, and family members. The complications of the tracheostomy, which frequently caused suffering to the patients, parents or caregivers and required frequent visits to a doctor, were documented, such as tracheostomy block, excessive mucus production, infection at the stoma, granulations tissue at the stoma, haemorrhage, and ugly neck scar. A questionnaire related to quality of life was completed by each caregiver, focusing on the physical symptoms of the patients, the social impact including the financial issues of medical visits, and quality of life. The social impact/issues or social setbacks related to the tracheostomy tube in the neck were documented, such as adjustment to the social environment, interference with professional voice use or helplessness in using a proper voice within the family or with friends, acceptance to near and dear ones, and having to frequently clean the tube.

\section{RESULTS}

Out of 24 patients with permanent tracheostomy, 15 were male and nine were female. The ages ranged from 3 years to 18 years, with mean age of 11.25 years. The youngest child in this study was three years old, who underwent tracheostomy for stridor due to bilateral vocal 
fold palsy (Fig. 1). The indications for tracheostomy in this study were laryngotracheal stenosis, bilateral vocal fold palsy, severe laryngomalacia, and postoperative total thyroidectomy (Table 1). Most common aetiology in this study was laryngotracheal stenosis $(45.83 \%)$ followed by bilateral vocal fold palsy $(29.16 \%)$, post-operative total thyroidectomy $(8.33 \%)$, trauma to the neck $(8.33 \%)$, severe laryngomalacia $(4.16 \%)$, and post-operative laryngectomy (4.16\%). At the time of examination, the patients had worn their tracheostomy tubes for more than one year, and the longest for five years.

Apart from complications of the tracheostomy itself, we identified several setbacks among the patients who underwent tracheostomy. Different setbacks due to tracheostomy included being unable to speak with a clear voice, family reluctance to accept the patient with a tracheostomy, problems of social integration and rehabilitation, and professional voice use. Informing about permanent tracheostomy was a rude shock to the patient and the patient's relatives, especially spouses, in all the cases. The euphoria after relief from the primary disease quickly disappears and is replaced by despair and misery. Parents and other relatives are terrified, and their frequency of visits continues to reduce. The constant question was, when will the tracheostomy tube be removed? Here, all the parents/caregivers/relatives asked the same question: when will the tracheostomy be removed from the neck of our child. This exposes their reluctance to take their child home with the tube.

Medical issues associated with tracheostomy are excessive mucus production through tracheostomy tube (70.83\%), infections at the stoma (33.33\%), tube blockage $(25 \%)$, formation of granulations at the stoma site $(16.66 \%)$, and maggots affecting the stoma site in one case $(4.16 \%)$ (Table 2$)$. The negative social issues associated with the tracheostomy tube in this study were reluctance to take care of family members (58.33\%), inability to communicate $(54.16 \%)$, difficulty with professional use of voice $(45.83 \%)$, and problems with social integration (41.66\%) (Table 3). In this study, 13 patients were unable to communicate to family members $(54.16 \%)$ whereas rest used a speaking tube for voice production. In this study 11 patients were professional voice users, and they lost their voice. One emotional issue associated with tracheostomy was when would the tracheostomy tube be removed, in all cases (100\%). The medical or surgical issues associated with a tracheostomy tube were promptly treated when they arose. Excessive production of mucus often responds to antihistamines and corticosteroids. The stoma infections were treated with antibiotics as per culture and sensitivity report. Granulations at the stoma were scraped or curetted from time to time, and sometimes chemicals such as silver nitrate were used, which acted as chemical cautery. Tube blockage was cleared by regular suction cleaning and tube changing. Parents/caregivers were trained about details of the inner, outer, and
TABLE 1. Indications for permanent tracheostomy in this study

\begin{tabular}{|l|c|c|}
\hline Indications & $\begin{array}{c}\text { Number } \\
\text { of patients }(n)\end{array}$ & $\begin{array}{c}\text { Percentage } \\
(\%)\end{array}$ \\
\hline Laryngotracheal stenosis & 11 & 45.83 \\
\hline Bilateral vocal fold palsy & 7 & 29.16 \\
\hline Post-op total thyroidectomy & 2 & 8.33 \\
\hline Severe laryngomalacia & 1 & 4.16 \\
\hline Trauma to the neck & 2 & 8.33 \\
\hline $\begin{array}{l}\text { Post-operative laryngectomy } \\
\text { patient }\end{array}$ & 1 & 4.16 \\
\hline
\end{tabular}

TABLE 2. Medical complications associated with tracheostomy tube

\begin{tabular}{|l|c|c|}
\hline Medical complications & $\begin{array}{c}\text { Number } \\
\text { of patients }(n)\end{array}$ & $\begin{array}{c}\text { Percentage } \\
(\%)\end{array}$ \\
\hline Excessive production of secretion & 17 & 70.83 \\
\hline Infections at the stoma & 8 & 33.33 \\
\hline Tube block & 6 & 25 \\
\hline Granulations at stoma & 4 & 16.66 \\
\hline Maggots at the stoma & 1 & 4.16 \\
\hline
\end{tabular}

TABLE 3. Social issues associated with tracheostomy tube

\begin{tabular}{|l|c|c|}
\hline Social issues & $\begin{array}{c}\text { Number } \\
\text { of patients }(n)\end{array}$ & $\begin{array}{c}\text { Percentage } \\
(\%)\end{array}$ \\
\hline Family reluctance & 14 & 58.33 \\
\hline Unable to communicate & 13 & 54.16 \\
\hline Loss of professional voice use & 11 & 45.83 \\
\hline Problems in social integration & 10 & 41.66 \\
\hline $\begin{array}{l}\text { Spouse/caregivers asking when } \\
\text { the tube will be removed }\end{array}$ & 24 & 100 \\
\hline
\end{tabular}

obturator of the tracheostomy tubes. None of the patients in this study presented with bleeding from stoma area except during scraping of curetting the granulations at the stoma region, which never created a problem.

\section{DISCUSSION}

Tracheostomy was introduced in medicine as a technique of relieving upper airway obstruction since the second century AD by Galen [5]. This surgical procedure has been used successfully to relieve upper respiratory tract obstruction and also helps to protect the lower respiratory tract and tracheobronchial toilet, and assists in ventilation - particularly in patients with prolonged unconsciousness. It is also often done preoperatively in major surgeries involving head and neck area, where breathing could be compromised [6]. Although rare, tracheostomy can be done in case of obstructive sleep apnoea syndrome if all other treatment options fail. In this study, the majority 
of the children's aetiologies for tracheostomy were laryngotracheal stenosis. Tracheostomy was primarily thought of as an emergency surgery for sudden upper airway obstruction caused by infectious aetiology like epiglottitis or diphtheria [7].

Tracheostomy is considered a safe surgical procedure, although it has its own minor and relatively rare major complications. The indications for tracheostomy include bypassing acute or chronic upper airway obstruction, which helps patients requiring long-term ventilatory support, prevents aspiration by giving access for tracheobronchial toilet, and prevents the formation of laryngotracheal stenosis in the case of prolonged intubation, as well as facilitation of weaning from a ventilator by removing ventilatory dead space. In the case of paediatric patients, the common indications for permanent tracheostomy include congenital and acquired airway stenosis, neurological conditions that need long-term ventilation or pulmonary toileting, bilateral vocal fold palsy, and infectious conditions compromising the upper airway [3].

In this study, one case of congenital severe laryngomalacia underwent tracheostomy for severe stridor. After thyroid surgery, breathing difficulty and stridor often indicate bilateral recurrent laryngeal nerve paralysis. In this study, two cases underwent tracheostomy for bilateral recurrent laryngeal nerve paralysis after total thyroidectomy. Other causes of upper airway obstructions after thyroid surgery include tracheomalacia, laryngeal oedema, or tracheal collapse due to long-standing compression of the trachea by a large goitre mass [8].

Tracheostomy may be of two types: temporary or permanent. In temporary tracheostomy, the tracheostomy tube stays in place for a few days to a few weeks, and the tube is finally removed at the time of resolution of the primary disease or when the airway obstruction due to such a condition is over. After removal of the tracheostomy tube, closure of the stoma is often done by use of a tight dressing or surgical closure. In the case of permanent tracheostomy, the tube stays in as long as the patient lives. This may be due to associated irreversible damage to the architecture of the larynx leading to inadequate respiratory function. Permanent tracheostomy children in our region often face social setbacks such as problems of adjusting to the social environment, family (parents/ siblings/relatives) reluctance to accept the child, interference with professional use of the voice, and often social withdrawal from near and dear ones.

Over the years, in clinical practice paediatric tracheostomy has been done by all types of surgeons, such as otolaryngologists and paediatric surgeons. The children who underwent permanent tracheostomy live with a tracheostomy tube in their neck for virtually all of their life. The attention of the patients and his or her surrounding people are often on the tube in the neck. Tracheostomy is usually associated with several complications such as tube block, excessive mucus secretion, stoma infections, polyp formation at the stoma, bleeding and laryngeal stenosis, and an ugly neck scar [3]. Decannulation of the tracheostomy tube is always the objective for surgeons and patients' families, but this is not possible in all cases. Studies have shown that the decannulation rates vary from approximately 35\% to $75 \%$ [9].

Although tracheostomy is a lifesaving surgery, it can cause emotional, financial, and psychological stress for parents and family members. A multidisciplinary team, such as surgeons, physicians, nurses, respiratory therapists, occupational therapists, speech therapists, nutritionists, and social workers, may be required for streamlining the care of paediatric patients with a tracheostomy tube. They are able to train the parents and caregivers. Patients with tracheostomy often have frequent appointments with physicians, during which trained staff or caregivers often accompany the patient in order to travel safely [10]. Stress, anxiety, strain, and burden are often associated with caregivers or parents. The socioeconomic status, resources available for caregivers, and stresses are important factors for managing a child with a tracheostomy. Prior to the discharge of tracheostomy patients, the caregivers or parents should be trained for the routine care of the tracheostomy tube. In particularly, they must be trained to identify the problems like the requirement of suctioning, breathing difficulties, and mucus plugging. The caregivers must be prepared to change the tracheostomy tube easily and efficiently. They should also be trained for cardiopulmonary resuscitation [11]. At home, certain things should be available close to the paediatric patient, such as a separate set of tracheostomy tubes, suction catheter, sterile saline, portable suction machines, humidifiers, and an oxygen saturation monitor [12]. All these must be in place and properly functioning.

The primary aim of parents or caregivers is to provide or help the children to take care of the tracheostomy in the home. Psychological preparation of parents is vital to them in order to provide optimum care. The stress may be intense when the patient is bedridden or fully dependent or sick, requiring mechanical ventilation, where the tracheostomy will add an extra layer of stress [13]. Social isolation, coping strategies, respiratory care, and emotional and physical outcomes after tracheostomy are important factors for creating anxiety among parents or caregivers [14]. Caregivers should be aware of concerns, such fear of separation, disfigurement, loss of loved ones, pain, and loss of autonomy [1]. The emotional and physical health of caregivers can affect the wellbeing of children with a tracheostomy [1].

Informing parents regarding permanent tracheostomy is often a rude shock to them and to their family members. The euphoria of the discharge of the child from the hospital quickly disappears and is replaced by despair and misery. Parents and relatives be terrified and their frequency of visits will continue to decline. They usually ask the question: when will be the tracheostomy tube will 
be removed from my child? In this study, parents/family members/relatives of all the participating children (100\%) were asking same questions about when the tube will be removed from the neck. They show reluctance to take their child home with tracheostomy tube. These types of rejection of the patients can be removed by rounds of discussion with doctors and family. The concerned members related to the patients are educated with a focus on benefits, complications, and explaining why the tracheostomy tube may not be removed or decannulated, as well as how to best care for the tracheostomy tube. In this study, the oldest patient or his/her parent was usually invited to share his/her experience and provide encouragement to the other patients and their relatives. Teaching patients, spouses, and relatives to change the tracheostomy tube becomes easier after a meeting/conference with doctors and the patient and his or her relatives. The parent or caregiver is sufficiently trained to master inner tube changing before discharge from the hospital [15].

A tracheostomy tube in the neck constitutes a social stigma and leads to anxiety in social interactions. It is not only cosmetically unattractive but also scarring in some communities. There are a number of costumes devised for preventing this social embarrassment by concealing the tracheostomy tube. Sometimes a high neck collar bib is designed to conceal the tracheostomy tube and the tapes attached to the tube. These are made of several colours in order to match any particular clothing worn by the child. The tracheostomy tube can be concealed by using different fashionable mufflers around the neck with the help of very light non-airtight materials. Necklaces are also designed like jewellery to replace the conventional tape of the tracheostomy tube. Management of social stigma related to tracheostomy is more difficult than other complications associated with a surgical procedure, so the family members, including parents, must be involved in it.

\section{CONCLUSIONS}

Caring for paediatric patients with a tracheostomy tube at home is often challenging for parents or caregivers. Careful preparation and planning are key to a successful transition to care in the home. Active participation for children with a tracheostomy tube will help the progression of health. For many, tracheostomy is a means to an end, which causes anxiety and stress to parents and caregivers. The problems due to tracheostomy in children are much greater than the complications due to surgery. The management of speech and its rehabilitation are always challenging and scarce among caregivers and parents. All efforts should be made for early tracheostomy in intensive care units, and optimum effort must be made to prevent bilateral recurrent laryngeal nerve paralysis during thyroidectomy surgery.

\section{DISCLOSURE}

The authors declare no conflict of interest.

\section{REFERENCES}

1. Hopkins $\mathrm{C}$, Whetstone S, Foster T, et al. The impact of paediatric tracheostomy on both patient and parent. Int J Pediatr Otorhinolaryngol 2009; 73: 15-20.

2. Wetmore RF, Handler SD, Potsic WP. Pediatric tracheostomy. Experience duringthe past decade. Ann Otol Rhinol Laryngol 1982; 91: 628-632.

3. Mahadevan M, Barber C, Salkeld L, et al. Pediatric tracheotomy: 17 year review. Int J Pediatr Otorhinolaryngol 2007; 71: 1829-1835.

4. Eisele DW, Smith RV. Complications in Head and Neck Surgery. $2^{\text {nd }}$ ed. Mosby Elsevier, Philadelphia 2009: 409416.

5. Amusa YB, Akinpelu VO, Fadiora SO, et al. Tracheostomy in surgical practice - experience in a Nigerian tertiary hospital. West Afr J Med 2004; 23: 3234.

6. Swain SK, Sahu MC, Choudhury J, et al. Tracheostomy among paediatric patients: Our experiences at a tertiary care teaching hospital in eastern India. Pediatr Pol 2018; 93: 312-317.

7. Carron JD, Derkay CS, Strope GL, et al. Pediatric tracheotomies: changing indications and outcomes. Laryngoscope 2000; 110: 10991104.

8. Bailey BJ, Johnson JT. Head and Neck Surgery - Otolaryngology. $4^{\text {th }}$ ed. Lippincott Williams and Wilkins, Philadelphia 2006: 663.

9. Ogilvie LN, Kozak JK, Chiu S, et al. Changes in pediatric tracheostomy 1982-2011: a Canadian tertiary children's hospital review. J Pediatr Surg 2014; 49: 1549-1553.

10. Douglas CM, Poole-Cowley J, Morrissey S, et al. Paediatric tracheostomy - a 11 year experience at a Scottish paediatric tertiary referral centre. Int J Pediatr Otorhinolaryngol 2015; 79: 1673-1676.

11. Flynn AP, Carter B, Bray L, et al. Parents experiences and views of caring for a child with a tracheostomy: a literature review. Int J Pediatr Otorhinolaryngol 2013; 77: 1630-1634.

12. Swain SK, Behera IC, Sahu MC. Bedside open tracheostomy at intensive care unit - Our experiences of 1000 cases at a tertiary care teaching hospital of eastern India. Egypt J Ear Nose Throat Allied Sci 2017; 18: 49-53.

13. Adoga AA, Ma'na ND. Indications and outcome of pediatric tracheostomy: results from a Nigerian tertiary hospital. BMC Surg 2010; 10: 2

14. Kirk S, Glendinning C, Callery P. Parent or nurse? The experience of being the parent of a technology-dependent child. J Adv Nursing 2005; 51: 456-464.

15. Swain SK, Das A, Behera IC, Bhattacharyya B. Tracheostomy among pediatric patients: A review. Indian J Child Health 2018; 9: $557-561$. 